\title{
The effect of fearful faces on the attentional blink is task dependent
}

\author{
TIMo STEIN \\ Ludwig-Maximilians-Universität, Munich, Germany \\ and Princeton University, Princeton, New Jersey \\ Jan Zwickel, Johanna RitTer, ANd Maria Kitzmantel \\ Ludwig-Maximilians-Universität, Munich, Germany \\ AND \\ Werner X. SCHNEIDER \\ Ludwig-Maximilians-Universität, Munich, Germany \\ and Universität Bielefeld, Bielefeld, Germany
}

\begin{abstract}
In a set of three rapid serial visual presentation experiments, we investigated the effect of fearful and neutral face stimuli on the report of trailing scene targets. When the emotional expression of the face stimuli had to be indicated, fearful faces induced a stronger attentional blink $(\mathrm{AB})$ than did neutral faces. However, with identical physical stimulation, the enhancement of the $\mathrm{AB}$ by fearful faces disappeared when participants had to judge the faces' gender. If faces did not have to be reported, no AB was observed. Thus, fearful faces exhibited an effect on the AB that crucially depended on the observer's attentional set. Hence, the AB can be influenced by an emotional T1 when $\mathrm{T} 1$ has to be reported, but this influence is modulated by task context. This result indicates a close connection between temporal attention and emotional processing that is modulated by task context.
\end{abstract}

In cluttered and rapidly changing visual environments, stimuli compete for limited processing resources. Selective attention resolves competition by facilitating processing of visual information that is behaviorally relevant at the expense of irrelevant input (Desimone \& Duncan, 1995). Both endogenous factors related to current goals or tasks and exogenous signals, such as sensory salience of external input, can exert control over the deployment of attentional resources and thereby influence perceptual processing (Kastner \& Ungerleider, 2000). Similarly, emotionally valenced, and in particular negative, stimuli that are exceptionally important for the survival of the organism appear to bias the competition for processing resources by attracting attention more effectively and more readily than do unemotional stimuli (Pessoa, Kastner, \& Ungerleider, 2002).

Most studies of visual attention have addressed the question of how multiple stimuli are processed when they are presented simultaneously in a single spatial array. However, even seemingly simple everyday activities like driving a car necessitate attentional selection in time. Temporal attentional processes are typically studied by requiring participants to select targets from a series of distractor stimuli presented at a rapid rate, which is called rapid serial visual presentation (RSVP; Potter \& Levy, 1969; Raymond, Shapiro, \& Arnell, 1992). The most prominent
RSVP paradigm is the attentional blink (AB; Raymond et al., 1992), in which two targets are placed in a sequence of distractor items. If both targets (T1 and T2) must be reported, identification or detection of T1 interferes with the processing of T2 for periods lasting several hundred milliseconds. Correct report of $\mathrm{T} 2$ is poorest at short temporal lags between the two target items and improves with increasing lags, whereas accuracy in report of $\mathrm{T} 1$ is affected only minimally by lag duration (see, e.g., Chun \& Potter, 1995; Raymond et al., 1992; Shapiro, Raymond, \& Arnell, 1994). Importantly, T2 performance usually shows no interference from $\mathrm{T} 1$ presentation in conditions in which $\mathrm{T} 1$ is ignored. Hence, reduced T2 accuracy at short lags is not due to sensory factors (Raymond et al., 1992) but, instead, reflects attentional demands associated with $\mathrm{T} 1$ processing, which leaves few resources available to process T2 (e.g., Chun \& Potter, 1995; Jolicœur \& Dell'Acqua, 1998; Shapiro et al., 1994).

Recent research has demonstrated that emotion-laden to-be-ignored T1 stimuli that were not to be reported nonetheless led to a deterioration of T2 performance at short lags (Arnell, Killman, \& Fijavz, 2007; Barnard, Ramponi, Battye, \& Mackintosh, 2005; Most, Chun, Widders, \& Zald, 2005; Most, Smith, Cooter, Levy, \& Zald, 2007; Smith, Most, Newsome, \& Zald, 2006). For instance, aversive pictures interfere with target report (Most

T. Stein, tstein@princeton.edu 
et al., 2005). The same holds true for positively arousing pictures (Most et al., 2007) and for aversively conditioned neutral pictures (Smith et al., 2006). Similar but smaller effects have been obtained using emotionally arousing words instead of pictures (Arnell et al., 2007; Barnard et al., 2005). Thus, to-be-ignored emotional stimuli seem to reliably capture temporal attention and interfere with target processing.

Most current explanations of the $\mathrm{AB}$ share a high degree of convergence by stating that a manipulation of $\mathrm{T} 1$ processing should exhibit an effect on T2 performance. However, only a few AB studies have examined the effect of emotional T1 picture stimuli on T2 accuracy when T1 had to be reported. De Jong and Martens (2007) inserted two upright happy or angry faces in an RSVP series of rotated neutral distractor faces and instructed observers to indicate both the number of perceived upright emotional faces and their emotional expression. In their study, the presentation of an angry face as the T1 did not alter T2 performance. Similarly, Arend and Botella (2002) did not find emotional words presented as to-be-reported T1 stimuli to influence T2 identification. In contrast, emotional words as the $\mathrm{T} 1$ exhibited a beneficial effect on $\mathrm{T} 2$ recognition, but only in high-trait-anxiety individuals.

The discrepancy between studies that found that tobe-ignored emotional stimuli induced an $\mathrm{AB}$ and studies that found no deterioration of $\mathrm{T} 2$ accuracy even when a response to the emotional stimulus was required could be due to the specific type of stimuli used in these studies. For instance, De Jong and Martens (2007) always presented happy or angry faces as T1 and T2 stimuli and found T2 performance to depend on the congruency between the emotions displayed by both stimuli. In contrast, in studies showing attentional capture by emotional pictures as to-be-ignored T1 stimuli (Most et al., 2005; Most et al., 2007; Smith et al., 2006), participants were required to report a neutral T2 stimulus, such as the orientation of a rotated scene picture.

Here, we attempted to shed some light on these conflicting results by keeping the stimulus materials constant across a series of three experiments, which varied only with regard to the specific task on the T1 stimulus. We examined how fearful and neutral faces preceding a scene target affected $\mathrm{T} 2$ performance. In Experiment 1, participants had to explicitly judge the facial expression of T1 stimuli; thus, the face pictures were task relevant, and attention was directed to their emotional expression. In Experiment 2, participants had to judge T1's gender; hence, the face pictures were task relevant, but attention was not explicitly directed to their emotional expression. In Experiment 3, T1 was task irrelevant and served as the critical distractor item to evaluate potential attentional capture.

If the presentation of a to-be-ignored fearful face as T1 captured attention, an AB should be observed in Experiment 3. Furthermore, we hypothesized that $\mathrm{T} 2$ performance would be modulated by the task relevance of the face presented as T1. If participants had to report T1, it should receive more attentional resources at the expense of $\mathrm{T} 2$ processing, leading to a larger $\mathrm{AB}$ in Experiments 1 and 2 than in Experiment 3. Moreover, the effect of fearful faces on the $\mathrm{AB}$ magnitude might be amplified by directing attention to their facial expression. Therefore, fearful faces might induce a stronger $\mathrm{AB}$ in Experiment 1, in which the participants' attention was directed to the faces' expression.

\section{METHOD}

\section{Participants}

In each of the three experiments, 14 participants took part (Experiment 1,6 female, mean age $=25.1$ years; Experiment 2,7 female, mean age $=25.2$ years; Experiment 3,9 female, mean age $=$ 23.9 years), who were compensated with course credit or were paid. All of the participants had normal or corrected-to-normal vision and reported no history of neurological disorder.

\section{Apparatus and Stimuli}

The participants viewed the monitor in a dimly lit room at a free viewing distance of approximately $55 \mathrm{~cm}$. All stimuli constituting the RSVP series were grayscale photographs (subtending $17.0^{\circ} \times$ $17.0^{\circ}$ of visual angle) presented on a gray background. The NimStim face stimulus set (Tottenham et al., in press) was used in order to generate three different pools of T1 stimuli. Two photographs, depicting a neutral and a fearful facial expression, respectively, were taken from each of 12 selected actors ( 6 female), yielding a pool of 12 neutral T1 face stimuli and a pool of 12 fearful T1 face stimuli. The distinction between neutral and fearful faces was verified by ratings after the experiment (see Table 1). The third T1 stimulus pool contained 96 scrambled versions of the neutral and fearful T1 face stimuli, referred to as the face-absent condition. The $\mathrm{T} 2$ stimuli were drawn from two pools of 96 indoor and 96 outdoor scenes. The scene photographs were copyright-released images selected from the Internet. The indoor scene photographs depicted rooms such as bathrooms, bedrooms, children's rooms, kitchens, or living rooms. The outdoor scene photographs depicted houses, junctions, residential streets, or public buildings. Neither indoor nor outdoor scene photographs contained humans or animals. Distractor items were 192

Table 1

Face Ratings for All Three Experiments

\begin{tabular}{|c|c|c|c|c|c|c|c|c|c|c|c|c|}
\hline & \multicolumn{6}{|c|}{ Neutral Faces } & \multicolumn{6}{|c|}{ Fearful Faces } \\
\hline & \multicolumn{2}{|c|}{ Valence } & \multicolumn{2}{|c|}{ Arousal } & \multicolumn{2}{|c|}{ Fearfulness } & \multicolumn{2}{|c|}{ Valence } & \multicolumn{2}{|c|}{ Arousal } & \multicolumn{2}{|c|}{ Fearfulness } \\
\hline & $M$ & $S D$ & $M$ & $S D$ & $M$ & $S D$ & $M$ & $S D$ & $M$ & $S D$ & $M$ & $S D$ \\
\hline Experiment 1 & 4.10 & 0.33 & 2.22 & 0.19 & 1.73 & 0.12 & 2.17 & 0.27 & 5.27 & 0.44 & 5.62 & 0.43 \\
\hline Experiment 2 & 4.27 & 0.46 & 2.10 & 0.42 & 1.51 & 0.40 & 2.48 & 0.37 & 5.58 & 0.56 & 5.56 & 0.59 \\
\hline Experiment 3 & 3.59 & 0.44 & 1.80 & 0.30 & 1.71 & 0.37 & 1.97 & 0.47 & 5.70 & 0.58 & 5.66 & 0.60 \\
\hline
\end{tabular}

Note-After completing the experiment, the participants rated each fearful and neutral face for valence (from 1, negative, to 7, positive), arousal (from 1, unstimulating, to 7, very stimulating), and fearfulness (from 1, not fearful, to 7 , very fearful). In all three experiments, participants rated fearful faces to be significantly more negative and more arousing and as expressing more fearfulness compared with neutral faces (all $p \mathrm{~s}<.0001$ ). 
scrambled versions of these indoor and outdoor scenes. Scrambled versions of faces and scenes were created by dividing each image into 121 squares and randomly recomposing them.

\section{Design and Procedure}

Each trial started with a 1.5 -sec presentation of a black fixation cross in the middle of the screen, followed by the sequential presentation of 13 stimuli, 2 targets (T1 and T2) and 11 distractors (see Figure 1). Each item in the series was presented for $67 \mathrm{msec}$ and was immediately followed by the trailing item. The series always started with 1 distractor item, and the T2 stimulus was drawn at the tenth position followed by 3 masking distractor items. There were eight lags between $\mathrm{T} 1$ and $\mathrm{T} 2$, ranging from lag 1 (no intervening distractor items, stimulus onset asynchrony [SOA] $=67 \mathrm{msec}$ ) to lag 8 (seven intervening distractor items, $\mathrm{SOA}=536 \mathrm{msec}$ ). At the end of the series, the participants were prompted to recall $\mathrm{T} 1$ and subsequently T2 (Experiments 1 and 2) or only to report T2 (Experiment 3). Participants were informed that their responses should be made as accurately as possible, without speed pressure. In Experiment 1 , responses to T1 were made with the left hand by typing "1" for "face absent," "2" for "neutral face," and "3" for "fearful face." In Experiment 2, the participants had to respond to T1 by typing " 1 " for "face absent," "2" for "female face," and " 3 " for "male face" with the left hand. T2 responses were made with the right hand by typing " 8 " for "indoor scene" and " 9 " for "outdoor scene." Feedback was given immediately after each response had been executed.

Within each testing session, there were six blocks consisting of 144 trials each: Each combination of three conditions (face absent, neutral, or fearful face) $\times$ eight lags $\times$ two T2 categories (indoor or outdoor scene) occurred for each trial with equal probability. The identities of the T1 and T2 stimuli were selected at random without replacement from their respective pools for each trial, and the trial order was randomized.

\section{RESULTS}

\section{Experiment 1}

Overall, T1 accuracy was $97.4 \%$ for the face-absent condition, $95.6 \%$ for neutral faces, and $93.8 \%$ for fearful faces. A one-way repeated measures ANOVA with the factor T1 condition showed that accuracy differed between $\mathrm{T} 1$ conditions $\left[F(2,26)=12.82, p<.0001, \eta_{\mathrm{p}}^{2}=\right.$ $.50]$. Bonferroni-corrected $t$ tests ( $p$ values are reported as Bonferroni-corrected by the number of tests) revealed that the absence of a face was reported more accurately than were fearful faces $\left[t(13)=6.39, p_{3}<.0005\right]$. Performance for neutral faces did not differ from that for the face-absent condition or for fearful faces $[t(13)=-2.22$, $p_{3}=.134$, and $t(13)=2.44, p_{3}=.089$, respectively]. In the face-absent condition, $56.4 \%$ of the errors resulted from confusions with neutral faces. In the neutral condition, $69.9 \%$ of the errors were reports of fearful faces, and in the fearful condition, $86.5 \%$ of the errors were confusions with neutral faces.
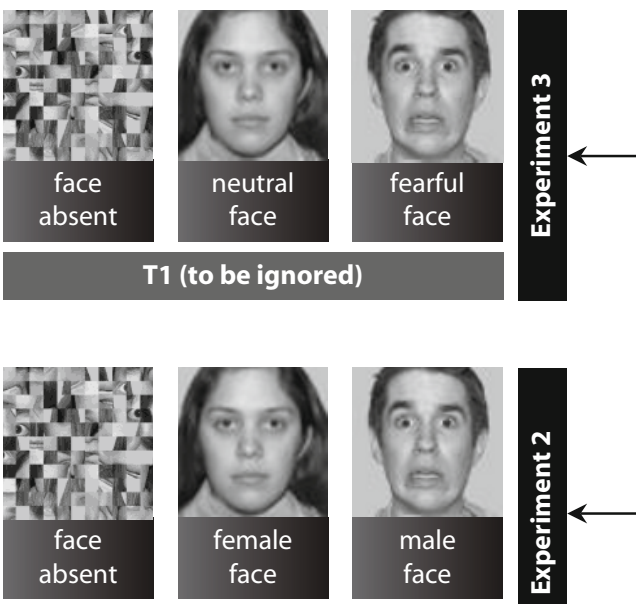

T1 (gender judgment)
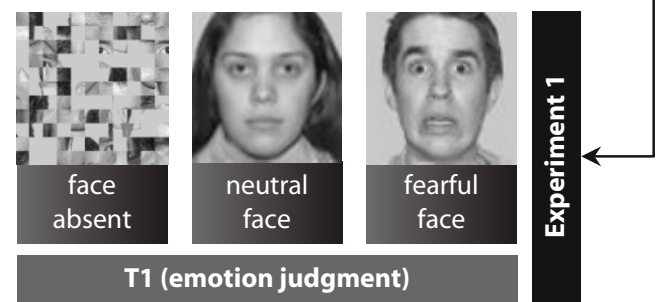

T1 (emotion judgment)

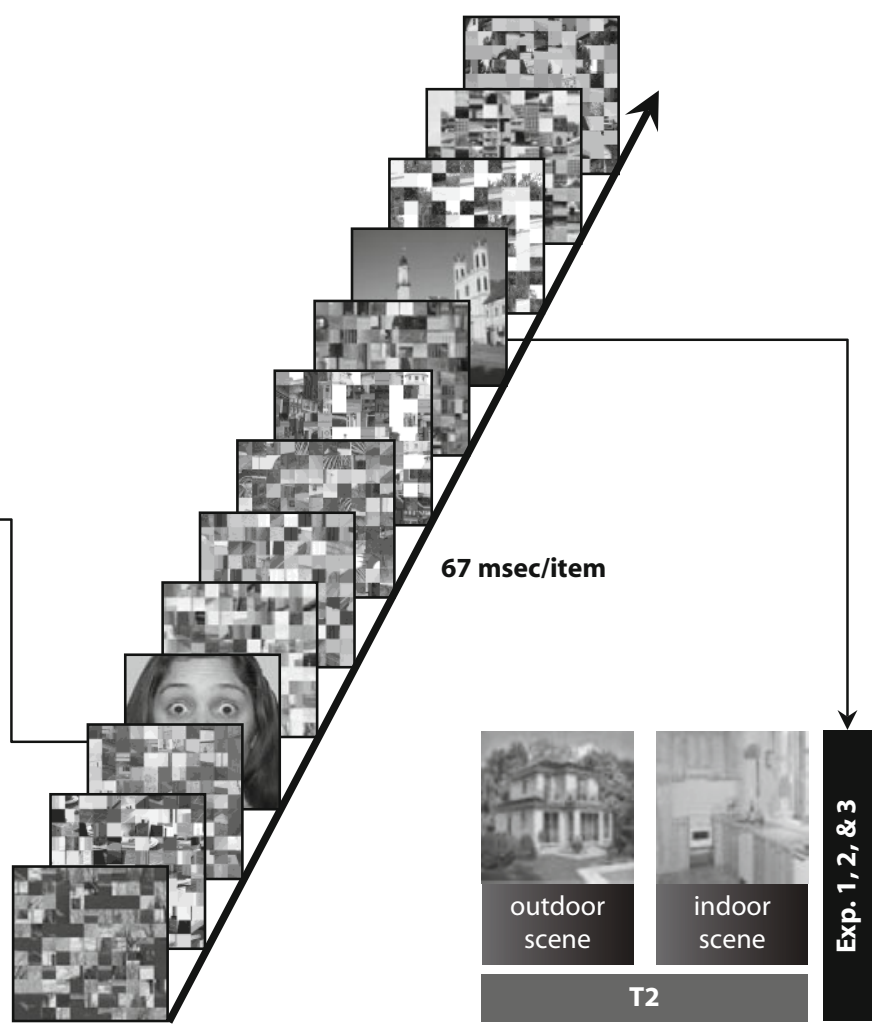

Figure 1. Experimental design and schematic of one example trial. Physical stimulation was identical for all three experiments. T1 was either a scrambled (i.e., face absent), neutral, or fearful face. T2 could be an outdoor or an indoor scene. The RSVP series consisted of scrambled scene photographs. In all three experiments, observers had to indicate whether T2 was an outdoor or an indoor scene. The T1 task was varied between experiments. In Experiment 1, participants judged T1's facial expression (face absent, neutral face, or fearful face). In Experiment 2, observers indicated T1's gender (face absent, female face, or male face). In Experiment 3, T1 could be ignored. 


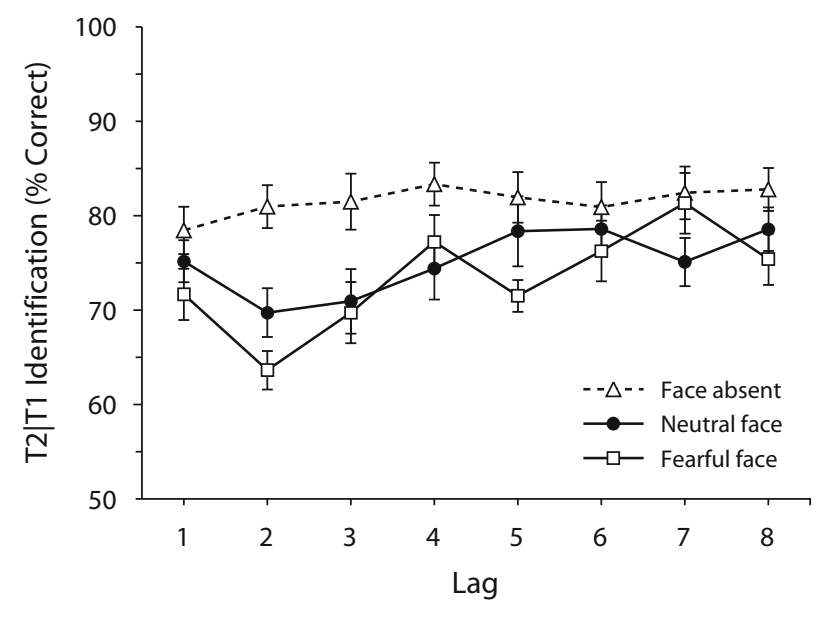

Figure 2. Mean percentage of accurate $\mathrm{T} 2$ report given correct T1 report at each T1-T2 lag in Experiment 1. Error bars represent standard errors of the means.

T2 performance was analyzed solely on the basis of trials in which T1 was accurately reported, since in T1incorrect trials, the source of error is unknown (see, e.g., Chun \& Potter, 1995). Figure 2 shows the percentages of correct identification of the T2 scene targets as a function of lag, presented separately for each condition. A twoway ANOVA on T2 performance yielded a reliable main effect of condition $\left[F(2,26)=19.31, p<.0001, \eta_{\mathrm{p}}^{2}=\right.$ $.60]$ and a significant main effect of lag $[F(7,91)=5.65$, $\left.p<.0001, \eta_{\mathrm{p}}^{2}=.30\right]$, as well as a significant interaction between condition and lag $[F(14,182)=2.56, p=.005$, $\left.\eta_{\mathrm{p}}^{2}=.17\right]$. Since there was no significant main effect of lag for the face-absent condition $[F(7,91)=0.75, p=$ $\left..629, \eta_{\mathrm{p}}^{2}=.06\right]$, subsequent analyses focused on the facepresent conditions only.

A two-way ANOVA on T2 performance for the two face-present conditions yielded a reliable main effect of lag $\left[F(7,91)=5.98, p<.0001, \eta_{\mathrm{p}}^{2}=.32\right]$, as well as a significant interaction between condition and lag $\left[F(7,91)=2.87, p<.01, \eta_{\mathrm{p}}^{2}=.18\right]$, but no significant main effect of condition $[F(1,13)=1.69, p=.217$, $\left.\eta_{\mathrm{p}}^{2}=.12\right]$. Bonferroni-corrected $t$ tests used to investigate differences in $\mathrm{T} 2$ performance at each lag revealed a significantly lower T2 accuracy in the fearful face condition than in the neutral face condition only at lag $2[t(13)=$ $\left.3.36, p_{8}<.05\right]$. In the majority of $\mathrm{AB}$ studies, the $\mathrm{AB}$ (i.e., reduced performance for $\mathrm{T} 2$ ) is accentuated at lag 2 . Thus, the present results show an enhancement of the AB by preceding fearful faces as compared with neutral faces.

\section{Experiment 2}

Overall T1 accuracy was $98.9 \%$ for the face-absent condition, $93.0 \%$ for neutral faces, and $91.8 \%$ for fearful faces. A one-way repeated measures ANOVA with the factor T1 condition showed that accuracy differed between T1 conditions $\left[F(2,26)=24.71, p<.0001, \eta_{\mathrm{p}}^{2}=.66\right]$. Bonferronicorrected $t$ tests revealed that the absence of a face was reported more accurately than were neutral and fearful faces $\left[t(13)=5.38, p_{3}<.0005\right.$, and $t(13)=5.11, p_{3}<.0005$, respectively]. Performance for neutral and fearful faces did not differ $\left[t(13)=1.96, p_{3}=.215\right]$. In the no-face condition, $62.8 \%$ of the errors resulted from confusions with neutral faces. In the neutral condition, $86.1 \%$ of the errors were reports of fearful faces, and in the fearful condition, $87.3 \%$ of the errors were confusions with neutral faces.

Figure 3 shows the percentages of correct T2 identification. A two-way ANOVA on T2 performance yielded a reliable main effect of condition $[F(2,26)=7.05, p<.005$, $\left.\eta_{\mathrm{p}}^{2}=.35\right]$ and a significant main effect of lag $[F(7,91)=$ $\left.14.30, p<.0001, \eta_{\mathrm{p}}^{2}=.52\right]$, as well as a significant interaction between condition and lag $[F(14,182)=2.58, p<$ $\left..05, \eta_{\mathrm{p}}^{2}=.17\right]$. Since there was no significant main effect of lag for the face-absent condition $[F(7,91)=1.65, p=$ $\left..131, \eta_{\mathrm{p}}^{2}=.11\right]$, subsequent analyses focused on the facepresent conditions only.

A two-way ANOVA on T2 performance for the two face-present conditions yielded a reliable main effect of lag $\left[F(7,91)=14.04, p<.0001, \eta_{\mathrm{p}}^{2}=.52\right]$. Neither the main effect of condition $[F(1,13)=0.52, p=.484$, $\left.\eta_{\mathrm{p}}^{2}=.04\right]$ nor the interaction between condition and lag $\left[F(7,91)=1.05, p=.405, \eta_{\mathrm{p}}^{2}=.07\right]$ reached significance. Hence, in Experiment 2, fearful faces did not induce a stronger $\mathrm{AB}$.

Comparing the results of Experiments 1 and 2, a threeway between-subjects ANOVA revealed a significant three-way interaction between experiment, condition (fearful or neutral), and lag $[F(7,182)=2.83, p<.01$, $\left.\eta_{\mathrm{p}}^{2}=.10\right]$. Post hoc $t$ tests revealed between-experiment effects for the difference between the neutral and the fearful face condition at lag $2[t(26)=2.37, p<.05]$ but not at lag $1[t(26)=-0.87, p=.392]$. Thus, it appears that task-relevant fearful faces can only trigger an enhanced $\mathrm{AB}$ when participants are explicitly required to focus their attention on the emotional expression.

\section{Experiment 3}

Figure 4 shows the percentages of correct T2 identification. A two-way ANOVA on T2 performance yielded

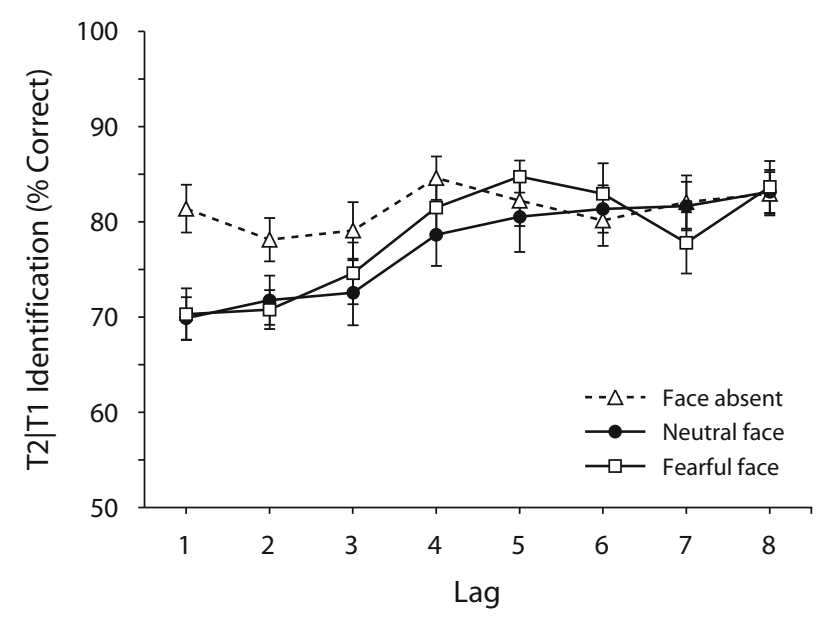

Figure 3. Mean percentage of accurate $\mathrm{T} 2$ report given correct T1 report at each T1-T2 lag in Experiment 2. Error bars represent standard errors of the means. 
neither a main effect of condition $[F(2,26)=1.37, p=$ $\left..272, \eta_{\mathrm{p}}^{2}=.10\right]$ nor a main effect of $\operatorname{lag}[F(7,91)=1.51$, $\left.p=.215, \eta_{\mathrm{p}}^{2}=.10\right]$. Crucially, the interaction between condition and lag $\left[F(14,182)=1.97, p=.080, \eta_{\mathrm{p}}^{2}=.13\right]$ did not reach statistical significance. These results indicate that if faces were not task relevant, they did not automatically capture and hold attention, regardless of their emotional expression.

\section{DISCUSSION}

The results of the present study are threefold. First, fearful faces induced a stronger $\mathrm{AB}$ than did neutral faces when faces were task relevant and attention was directed to the facial expression. Second, with identical physical stimulation, the enhancement of the $\mathrm{AB}$ by fearful faces disappeared when participants' attention was directed to a nonemotional facial feature (i.e., the faces' gender). Third, to-be-ignored faces did not capture and hold attention, regardless of their emotional content.

In theoretical accounts of the $\mathrm{AB}$, researchers have assumed that performance during short lags deteriorated because items closely succeeding $\mathrm{T} 1$ were forced to compete for attentional resources already engaged by $\mathrm{T} 1$ (e.g., Chun \& Potter, 1995; Jolicœur \& Dell'Acqua, 1998). The results from Experiment 1 suggest that fearful faces attract more attentional resources, leaving less processing capacity for a $\mathrm{T} 2$ appearing at lag 2 . This result is in line with recent findings suggesting that observers take longer to disengage spatial visual attention from threat-related stimuli, such as fearful faces, than from neutral stimuli (Fox, Russo, Bowles, \& Dutton, 2001; Georgiou et al., 2005). Interestingly, fearful faces, as compared with neutral and happy faces, evoke stronger activity in occipitotemporal visual cortex (Pessoa, McKenna, Gutierrez, \& Ungerleider, 2002). Pessoa, Kastner, and Ungerleider (2002) speculated that this result could constitute a mechanism of prioritizing emotional information at the expense of reduced processing capacities for neutral stimuli. Possibly, a similar mechanism formed the basis of reduced performance for scenes when they were closely preceded by fearful faces whose emotional expression had to be reported.

Experiment 2 demonstrated that the effect of fearful faces on the $A B$ is subject to the manipulation of the observers' attentional set. When participants had to report a nonemotional facial feature (i.e., gender), fearful faces exhibited no deteriorating effect on subsequent scene identification. Thus, interference by fearful faces in the $\mathrm{AB}$ is not triggered independently of the participants' task set. This result provides evidence against proposals in which it is assumed that emotion-laden stimuli are processed involuntarily and automatically (Vuilleumier, Armony, Driver, \& Dolan, 2001) and supports the idea that attentional resources are required for processing stimulus valence (Pessoa, Kastner, \& Ungerleider, 2002; Pessoa, McKenna, et al., 2002; Silvert et al., 2007).

In Experiment 3, task-irrelevant faces did not affect T2 accuracy relative to the face-absent condition. Showing that neutral faces do not capture temporal attention, the results of the present study replicated the results by Marois,

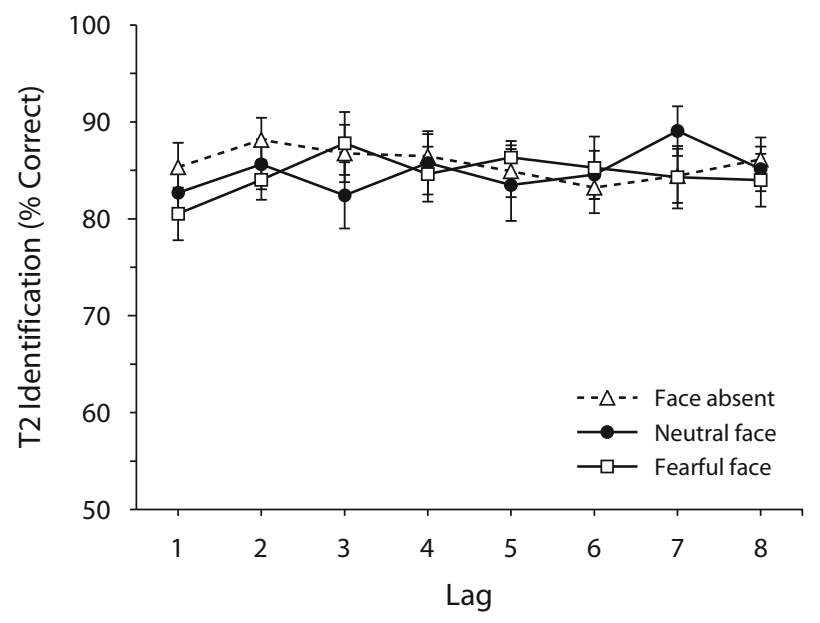

Figure 4. Mean percentage of accurate T2 report at each T1-T2 lag in Experiment 3. Error bars represent standard errors of the means.

Yi, and Chun (2004), who found no reduced scene detection performance in an $\mathrm{AB}$ paradigm when neutral faces could be ignored. Studies investigating the effect of neutral distractor stimuli in RSVP series have demonstrated that only distractor items precisely matching the participants' attentional set, as given by the target-defining feature, can trigger attentional capture (Folk, Leber, \& Egeth, 2002; Lamy, Leber, \& Egeth, 2004; Leblanc \& Jolicœur, 2005; Maki \& Mebane, 2006). In the present experiments, faces did not share features with the scene target and, therefore, did not induce attentional capture in Experiment 3. However, the finding of no interference from fearful faces on T2 performance is in marked contrast to studies reporting attentional capture by emotion-laden to-be-ignored stimuli (Arnell et al., 2007; Barnard et al., 2005; Most et al., 2005; Most et al., 2007; Smith et al., 2006).

A reason for this discrepancy could be the different stimulus materials used in the present experiments. In studies in which attentional capture by task-irrelevant emotional words inserted in a series of neutral words was found (Arnell et al., 2007; Barnard et al., 2005), T2 was defined only by its particular meaning (e.g., a job-related word or a color word). Therefore, observers possibly adopted the strategy of semantically processing all RSVP items, including the to-be-ignored emotional word, in order to be successful in identifying T2. This idea has recently received support from a study demonstrating that to-be-ignored emotional words at $\mathrm{T} 1$ led to a deterioration of T2 accuracy only when participants were required to process T2 semantically but not when perceptual or phonological processing of T2 was required (Huang, Baddeley, \& Young, 2008). Other studies revealing attentional capture by to-be ignored emotional stimuli have used highly disturbing (Most et al., 2005; Most et al., 2007) or fear-conditioned (Smith et al., 2006) stimuli that are probably more efficient in capturing temporal attention.

Thus, it appears that not all emotional stimuli automatically hold temporal attention. The effect of faces on the $\mathrm{AB}$ seems to be sensitive to the observer's task set. In this 
line, it has been demonstrated that fMRI repetition suppression for fearful faces is found only when they are task relevant, not when they are task irrelevant (Ishai, Pessoa, Bikle, \& Ungerleider, 2004).

In conclusion, fearful faces exhibit a stronger effect on the AB than do neutral faces, but this effect of emotional valence on attentional control depends crucially on the task set. Whether this conclusion on the relationship between emotion and attention generalizes to other kinds of emotional stimuli, such as dangerous animals or threatening scenes, or to other nonemotional but motivationally highly significant stimuli is an open empirical issue subject to further study.

\section{AUTHOR NOTE}

This research was funded by a grant from CoTeSys (Cognition for Technical Systems Cluster of Excellence) Project 125 and by Grant FOR 480 (TP4) from the German Research Foundation. Development of the NimStim Face Stimulus Set was overseen by Nim Tottenham and supported by the John D. and Catherine T. MacArthur Foundation Research Network on Early Experience and Brain Development. Please contact Nim Tottenham at tott0006@tc.umn.edu for more information concerning the stimulus set. Correspondence concerning this article should be sent to T. Stein, Department of Psychology, Princeton University, Green Hall, Princeton, NJ 08540 (e-mail: tstein@princeton.edu).

\section{REFERENCES}

Arend, I., \& Botella, J. (2002). Emotional stimuli reduce the attentional blink in subclinical anxious subjects. Psicothema, 14, 209-214.

Arnell, K. M., Killman, K. V., \& FiJavZ, D. (2007). Blinded by emotion: Target misses follow attention capture by arousing distractors in RSVP. Emotion, 7, 465-477.

Barnard, P. J., Ramponi, C., Battye, G., \& Mackintosh, B. (2005). Anxiety and the deployment of visual attention over time. Visual Cognition, 12, 181-211.

Chun, M. M., \& Potter, M. C. (1995). A two-stage model for multiple target detection in rapid serial visual presentation. Journal of Experimental Psychology: Human Perception \& Performance, 21, 109-127.

De Jong, P. J., \& Martens, S. (2007). Detection of emotional expressions in rapidly changing facial displays in high- and low-socially anxious women. Behaviour Research \& Therapy, 45, 1285-1294.

Desimone, R., \& Duncan, J. (1995). Neural mechanisms of selective visual attention. Annual Review of Neuroscience, 18, 193-222.

Folk, C. L., Leber, A. B., \& Egeth, H. E. (2002). Made you blink! Contingent attentional capture produces a spatial blink. Perception \& Psychophysics, 64, 741-753.

Fox, E., Russo, R., Bowles, R., \& Dutton, K. (2001). Do threatening stimuli draw or hold visual attention in subclinical anxiety? Journal of Experimental Psychology: General, 130, 681-700.

Georgiou, G. A., Bleakley, C., Hayward, J., Russo, R., Dutton, K., Eltiti, S., \& Fox, E. (2005). Focusing on fear: Attentional disengagement from emotional faces. Visual Cognition, 12, 145-158.

Huang, Y.-M., Baddeley, A., \& Young, A. W. (2008). Attentional capture by emotional stimuli is modulated by semantic processing. Journal of Experimental Psychology: Human Perception \& Performance, 34, 328-329.
Ishai, A., Pessoa, L., Bikle, P. C., \& Ungerleider, L. G. (2004). Repetition suppression of faces is modulated by emotion. Proceedings of the National Academy of Sciences, 101, 9827-9832.

Joliceur, P., \& Dell'AcQua, R. (1998). The demonstration of shortterm consolidation. Cognitive Psychology, 36, 138-202.

Kastner, S., \& Ungerleider, L. G. (2000). Mechanisms of visual attention in the human cortex. Annual Review of Neuroscience, 23, 315-341.

Lamy, D., Leber, A., \& Egeth, H. E. (2004). Effects of task relevance and stimulus-driven salience in feature-search mode. Journal of Experimental Psychology: Human Perception \& Performance, 30, 1019-1031.

Leblanc, E., \& Jolicceur, P. (2005). The time course of the contingent spatial blink. Canadian Journal of Experimental Psychology, 59, 124-131.

Maki, W. S., \& Mebane, M. W. (2006). Attentional capture triggers an attentional blink. Psychonomic Bulletin \& Review, 13, 125-131.

Marois, R., Yi, D.-J., \& Chun, M. M. (2004). The neural fate of consciously perceived and missed events in the attentional blink. Neuron, 41, 465-472.

Most, S. B., Chun, M. M., Widders, D. M., \& Zald, D. H. (2005). Attentional rubbernecking: Cognitive control and personality in emotion-induced blindness. Psychonomic Bulletin \& Review, 12, 654-661.

Most, S. B., Smith, S. D., Cooter, A. B., Levy, B. N., \& Zald, D. H. (2007). The naked truth: Positive, arousing distractors impair rapid target detection. Cognition \& Emotion, 21, 964-981.

Pessoa, L., Kastner, S., \& Ungerleider, L. G. (2002). Attentional control of the processing of neutral and emotional stimuli. Cognitive Brain Research, 15, 31-45.

Pessoa, L., McKenna, M., Gutierrez, E., \& Ungerleider, L. G. (2002). Neural processing of emotional faces requires attention. Proceedings of the National Academy of Sciences, 99, 11458-11463.

PotTer, M. C., \& LEVY, E. I. (1969). Recognition memory for a rapid sequence of pictures. Journal of Experimental Psychology, 81, 10-15.

Raymond, J. E., Shapiro, K. L., \& Arnell, K. M. (1992). Temporary suppression of visual processing in an RSVP task: An attentional blink? Journal of Experimental Psychology: Human Perception \& Performance, 18, 849-860.

Shapiro, K. L., Raymond, J. E., \& Arnell, K. M. (1994). Attention to visual pattern information produces the attentional blink in rapid serial visual presentation. Journal of Experimental Psychology: Human Perception \& Performance, 20, 351-371.

Silvert, L., Lepsien, J., Fragopanagos, N., Goolsby, B., Kiss, M., TAYLOR, J. G., ET AL. (2007). Influence of attentional demands on the processing of emotional facial expressions in the amygdala. NeuroImage, 38, 357-366.

Smith, S. D., Most, S. B., Newsome, L. A., \& Zald, D. H. (2006). An emotion-induced attentional blink elicited by aversively conditioned stimuli. Emotion, 6, 523-527.

Tottenham, N., Tanaka, J., Leon, A. C., McCarry, T., Nurse, M., Hare, T. A., ET AL. (in press). The NimStim set of facial expressions: Judgments from untrained research participants. Psychiatry Research.

Vuilleumier, P., Armony, J. L., Driver, J., \& Dolan, R. J. (2001). Effects of attention and emotion on face processing in the human brain: An event-related fMRI study. Neuron, 30, 829-841.

(Manuscript received March 12, 2008; revision accepted for publication July 20, 2008.) 